\title{
Effect of ursolic acid on obesity-induced insulin resistance in rat liver
}

\author{
Wang Ziwei ${ }^{1}$, Zhou $\mathrm{Na}^{1}$, Fang Shengbo ${ }^{2}$, Zhang Xin ${ }^{2 \star}$ \\ ${ }^{1}$ Department of Pharmacy, School and Hospital of Stomatology, Jilin University, ${ }^{2}$ Department of Pharmacy, First Hospital of Jilin \\ University, Changchun City, China
}

*For correspondence: Email: ms0746@163.com; Tel: +86-0431-85579358

Sent for review: 18 January 2018

Revised accepted: 22 April 2018

\begin{abstract}
Purpose: To determine the expression of protein tyrosine phosphatase-1B (PTP-1B) and insulin receptor substrate-2 (IRS-2) in the liver tissue of obesity-induced insulin-resistant rats.

Methods: Insulin resistance (IR) was induced in Wistar rats by placing them on a high fat diet for 6 weeks, and ursolic acid (UA) was administered. Metformin served as positive control drug. The rats were divided into 5 groups based on the treatments given: normal group, positive control group, metformin group, high-dose UA group, and low-dose UA group. The general conditions of the rats were assessed 4 and 8 weeks after the various treatments. Liver glycogen levels were measured, and liver histological examination carried out after tissue processing and staining with hematoxylin and eosin $(H$ \& E). Real-time polymerase chain reaction (RT-PCR) was employed for the determination of hepatic expressions of PTP-1B and IRS-2 mRNAs, while expressions of PTP-1B protein and IRS-2 protein, and phosphorylation of IRS-2 tyrosine were assayed by Western blotting.

Results: Liver glycogen levels were significantly increased in the UA-treated groups $(p<0.05)$. Moreover, UA provoked reductions in the expression of PTP-1B protein $(p<0.05)$, but up-regulated the expression of IRS-2 protein $(p<0.05)$, and enhanced IRS-2 tyrosine phosphorylation $(p<0.05)$.

Conclusion: These results suggest that UA mitigates IR through blockage of PTP-1B expression and up-regulation of the expression of IRS-2 mRNA. Therefore, PTP-1B is a potential target for the treatment of type 2 diabetes.
\end{abstract}

Keywords: Ursolic acid, Insulin resistance, Liver, Protein tyrosine phosphatase-1B, Insulin receptor substrate-2

\begin{abstract}
This is an Open Access article that uses a funding model which does not charge readers or their institutions for access and distributed under the terms of the Creative Commons Attribution License (http://creativecommons.org/licenses/by/4.0) and the Budapest Open Access Initiative (http://www.budapestopenaccessinitiative.org/read), which permit unrestricted use, distribution, and reproduction in any medium, provided the original work is properly credited.
\end{abstract}

Tropical Journal of Pharmaceutical Research is indexed by Science Citation Index (SciSearch), Scopus, International Pharmaceutical Abstract, Chemical Abstracts, Embase, Index Copernicus, EBSCO, African Index Medicus, JournalSeek, Journal Citation Reports/Science Edition, Directory of Open Access Journals (DOAJ), African Journal Online, Bioline International, Open-J-Gate and Pharmacy Abstracts

\section{INTRODUCTION}

Obesity is a risk factor for IR through endocrine, adipocytokine, inflammatory response and intracellular signaling pathways [1]. The liver is the main target organ for insulin action in the regulation of glycogen synthesis and glycogenolysis $[2,3]$. Insulin receptor substrate-2
(IRS-2) of the insulin signal cascade is closely related to hepatocyte insulin sensitivity [4]. The main physiological signal transduction pathway of insulin in the liver is IRS-2/phosphatidylinositol 3-kinase (PI-3K) [5]. Decreases in expressions of IRS-2 gene and disorders in phosphorylation affect the downstream signal delivery of PI-3K protein kinase $B(A k t)$, leading to IR. A member 
of the protein tyrosine phosphatase (PTP) family, protein tyrosine phosphatase-1B (PTP-1B) regulates intracellular substrates and tyrosine phosphorylation in conjunction with other members of the PTP family and protein tyrosine kinases, thereby inactivating signal molecules such as insulin receptor InsR) and insulin receptor substrate (IRs). Thus, PTP-1B regulates insulin signal transduction through blockage of the insulin signal cascade [6].

Ursolic acid (UA) is a triterpenoid compound present in natural plants, and one of the bioactive phytochemical components of Chinese traditional medicines used for treating diabetes. It inhibits transcription of genes related to phosphoenolpyruvate shuttle in hepatic gluconeogenesis, prevents $\beta$-cell apoptosis and enhances $\beta$-cell function $[7,8]$. In addition, UA enhances the phosphorylation of insulinmediated insulin receptor, protein kinase- $B$ and glycogen synthase kinase-3, and also enhances the transposition of insulin-to-glucose transporter-4 (GLUT4) in adipocytes $[9,10]$.

Metformin is a biguanide hypoglycemic agent which increases peripheral tissue glucose uptake and sensitivity to insulin while reducing peripheral tissue IR. Several investigations have reported the effect of UA on different tissues such as adipocytes, muscle, blood, L6 myotubes, and kidney [8,11-15]. However, the effect of UA on obesity-induced IR in the liver has not received much attention.

It has been demonstrated that the improvement of IR by UA is comparable to that of metformin. In the present study, the effect of UA on high fat diet-induced IR was investigated in a rat model by determination of hepatic expressions of PTP$1 \mathrm{~B}$ and IRS-2, and tyrosine phosphorylation in IRS-2, so as to provide experimental evidence for the prevention and treatment of diabetes mellitus.

\section{EXPERIMENTAL}

\section{Drugs and reagents}

Biosynthetic human insulin was product of Danish Novo Nordisk; UA was purchased from Shanghai Honghu Trade Co. Ltd, while metformin hydrochloride tablets were bought from Shanghai Xinyi Pharmacy. Trizol kit and Trizol reagent kits were products of Invitrogen, USA. Rabbit Anti-human PTP-1B cloning antibody, rabbit anti-human IRS-2 polyclonal antibody and IRS-2 phosphorylated tyrosine antibody were purchased from Abcam. Primer was obtained from Dawei Biotechnology Co., Ltd.
Brightening agent was from $E$ Healthcare; electrophoresis buffer and acrylamide were products of Shanghai Biyun Biotechnology Co., Ltd.), and RIPA buffer was purchased from Shanghai Biyun Days Biological Technology Co., Ltd. PVDF film was produced by Millipore.

\section{Ethical approval}

The present study received approval (approval no. 20180101) from the Ethical Committee of Department of Pharmacy of Jilin University Stomatological Hospital, and was carried out in compliance with Helsinki Declaration of 1964 as amended in 1996 [16].

\section{Rat groups and treatments}

Healthy male Wistar rats $(n=120,8$ weeks of age, body weight $180 \sim 200 \mathrm{~g}$ ) were provided by Shanghai Slack Experimental Animal Co., Ltd (certificate no. SYXK-2007-0003). A total of 24 rats were assigned to the normal group, while the remaining 96 rats comprised the positive control group and treatment groups (UA groups and metformin group). The normal group received basic diet provided by Animal Center of the Second Military Medical University. The other groups ( $n=24$ each) were given a high fat diet (consisting of basic diet, $10 \%$ lard oil, $1 \%$ cholesterol, $10 \%$ yolk powder and $0.1 \%$ bile salt) provided by Shanghai Slack Experimental Animal Co., Ltd. The rats were fed the high fat diet for 6 consecutive weeks to produce obesityinduced IR model. After 6 weeks, rats in UA groups were randomly divided into low and highdose UA groups (24 rats each) and treated with high fat diet containing $0.14 \%$ and $0.27 \% \cup A$, respectively. The metformin group was given 50 $\mathrm{mg}$ metformin/kg/day $[11,15]$.

\section{Morphological examination of liver tissues}

Liver tissues were processed for light microscopy, and sectioned and stained with H\&E. The stained sections were examined under the light microscope for pathological changes such as steatosis, fibrosis and inflammation.

\section{Determination of liver PTP-1B and IRS-2 mRNA}

Liver PTP-1B and IRS-2 mRNAs were determined by RT-PCR. Total RNA extraction from liver tissue was carried out using TRizol reagent in line with kit instructions. Forward and reverse primers were provided by Shanghai Daweike Company. The forward and reverse primers for PTP-1B were: 
Forward: 5'-aCC CTG TGC GGA AAT GCGGG3'

Reverse: 5'-GGC AGT CAG TCA ACC CCG GC$3^{\prime}$.

For IRS-2, the forward and reverse primers were:

Forward: 5'-TGC GAA CAG CCG TCG GTG AC3'

Reverse: 5'-GAC CGG TGA CGG CTG AAC GG-3'.

The forward and reverse primers for GAPDH were:

Forward: 5'-aGA ACA TCA TCC CTG CAT CC-3' Reverse: 5'-TGG ATA CAT TGG GGG TAG GA3'.

Real-time fluorescence quantitative PCR was performed after reverse transcription. The relative concentrations of PTP-1B, IRS-2 and GAPDH mRNAs were obtained from the standard curve. The expression of GAPDH was reflected by the ratio of relative concentration of PTP-1B, IRS-2 and GAPDH. Relative expression (RE) of mRNA calculated as in Eq 1.

$R E_{\text {mRNA }}=2^{-\Delta C T} \times 100$

where $\Delta C T=(C T$ value of target gene $)-(C T$ value of internal reference GAPDH).

\section{Expression of PTP-1B and IRS-2 protein and IRS-2 tyrosine phosphorylation levels}

After conventional methods of extraction of tissue proteins, polypropylene phthalamide gel electrophoresis and brightening agent were used for determination of the target protein concentration.

\section{Statistical analysis}

Data are expressed as mean $\pm S D$, and Student's $t$-test was used to compare between groups. SPSS 19.0 software was employed for statistical analysis. Statistical significance was assumed when $p<0.05$.

\section{RESULTS}

\section{Insulin sensitivity}

In line with homeostasis model, assessment insulin resistance (HOMO-IR) index for assessment of IR, the HOMA-IR index of the high-fat diet group $(0.82 \pm 0.22)$ was significantly higher than that of normal diet group $(0.65 \pm$ $0.11, t=3.20, p=0.00$; Figure 2) $[17,18]$. This demonstrates that insulin sensitivity was significantly decreased and that IR was successfully established after 6 weeks of exposure to high fat diet.

\section{Physical condition of the rats}

In the normal group, the rats had good mental state, normal appetite and clear skin. On the other hand, rats in treatment group were lethargic and docile, and had dull skin.

\section{Liver glycogen levels}

The levels of glycogen in the UA-treated groups were significantly higher than corresponding level in the normal control group $(p<0.05, p<0.01)$. The level of glycogen in high-dose UA group at week 8 was significantly higher than that of the metformin group $(p<0.01)$. These results are shown in Table 1.

Table 1: Effect of various treatments on liver glycogen levels (mean \pm SD)

\begin{tabular}{|c|c|c|c|}
\hline Group & Week & $\mathbf{n}$ & $\begin{array}{c}\text { Liver glycogen } \\
\text { level }\end{array}$ \\
\hline \multirow{2}{*}{ Normal } & 4 & 12 & $32.44 \pm 6.81$ \\
\hline & 8 & 12 & $36.63 \pm 2.98$ \\
\hline \multirow[b]{2}{*}{ Positive control } & 4 & 12 & $7.05 \pm 2.52^{\pi \pi}$ \\
\hline & 8 & 12 & $6.95 \pm 2.60$ \\
\hline \multirow[b]{2}{*}{ Metformin } & 4 & 12 & $12.89 \pm 2.71^{\wedge \Delta}$ \\
\hline & 8 & 10 & $12.46 \pm 1.73^{n \Delta \Delta}$ \\
\hline \multirow{2}{*}{ High-dose UA } & 4 & 12 & $17.91 \pm 2.80^{\pi \Delta \Delta}$ \\
\hline & 8 & 12 & $17.32 \pm 2.61^{\pi \times \Delta \Delta \Delta \Delta}$ \\
\hline \multirow{2}{*}{ Low-dose UA } & 4 & 12 & $15.38 \pm 2.63^{\Delta \Delta}$ \\
\hline & 8 & 12 & $15.14 \pm 2.43^{\wedge \wedge \Delta \Delta}$ \\
\hline \multicolumn{4}{|c|}{$\begin{array}{l}P<0.05 \text { and } p<0.01 \text {, compared to normal group; }{ }^{\Delta} p \\
<0.05 \text { and } \Delta \Delta p<0.01 \text {, compared to positive control } \\
\text { group; } p<0.05 \text { and }{ }_{p}{ }_{p}<0.01 \text {, compared to } \\
\text { metformin group }\end{array}$} \\
\hline
\end{tabular}

\section{Liver morphology}

There was no significant abnormality in the morphology of liver tissue in the normal group. In contrast, rats in the model group showed severe fatty degeneration and vacuoles in hepatocytes. Part of the liver cells showed diffuse necrosis, infiltration of inflammatory cells and slight fibrosis.

Treatment with UA produced some improvements in liver pathology, as evident in mild-to-moderate steatosis, decreased steatosis cells, normal hepatocellular morphology, decreased cytoplasmic lipid droplets, clear cytoplasm, absence of inflammatory cell infiltration and absence of fibrosis (Figure 1 and Figure 2). 


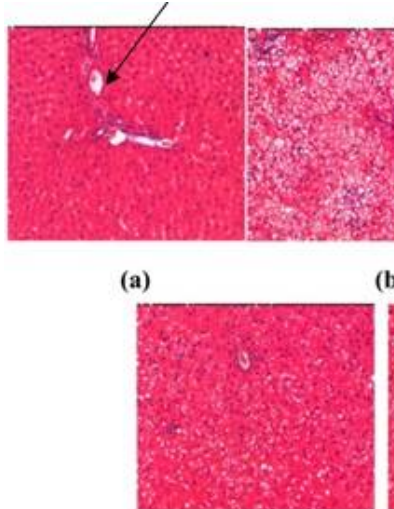

(d) (b)

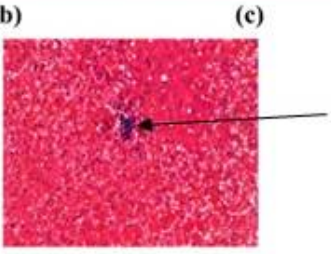

(e)
Figure 1: Liver morphology at week 4. (a) Normal group; (b) Positive control group; (c) Metformin group; (d) High-dose UA group; (e) low-dose UA group

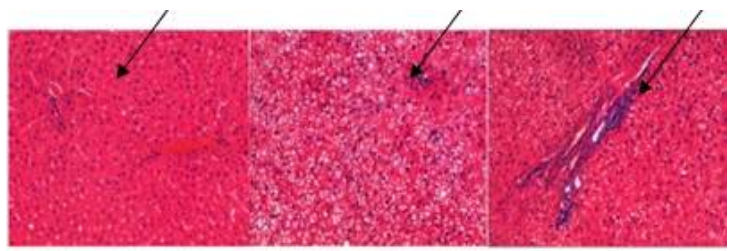

(a)

(c)

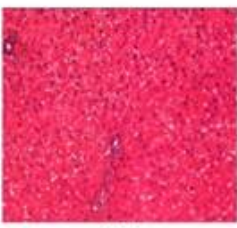

(d)

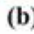

(b)

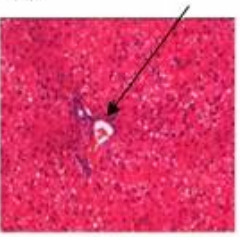

(e)
Figure 2: Liver morphology at week 8. (a) Normal group; (b) positive control group; (c) Metformin group; (d) High-dose UA group; (e) Low-dose UA group

\section{Expression of PTP-1 and IRS-2 mRNA in liver tissue}

Normal group had high expression of IRS-2 mRNA and low expression of PTP-1 mRNA, while positive control group had low expression of IRS-2 mRNA and high expression of PTP-1 mRNA. Thus, UA and metformin treatments increased the expressions of IRS-2 mRNA and reduced the expression of PTP-1B mRNA $(p<$ $0.01, p<0.05)$. These results are shown in Table 2.

\section{Expression of PTP-1B and IRS-2 protein in rat liver}

The results of western blot showed that the expression of PTP-1B protein in liver tissue of the positive control group was higher than that in normal control group, while the expression of IRS-2 protein was lower. However, after 4 and 8 weeks of UA exposure, the expression of PTP$1 \mathrm{~B}$ protein decreased while the expression of IRS-2 protein increased in the UA-treated groups. The effects produced by metformin were similar to those of UA (Figure 3 and Figure 4).
Table 2: Effect of UA and metformin on the expressions of PTP-1 and IRS-2 mRNA in liver tissue $($ mean $\pm S D, n=8$ )

\begin{tabular}{|c|c|c|c|}
\hline Group & Week & $\begin{array}{c}\text { PTP-1B mRNA } \\
\left(100 \times 2^{-\Delta C T}\right)\end{array}$ & $\begin{array}{c}\text { IRS-2 mRNA } \\
\left(100 \times 2^{-\Delta C T}\right)\end{array}$ \\
\hline & 4 & $0.22 \pm 0.02$ & $7.07 \pm 0.78$ \\
\hline Normal & 8 & $0.38 \pm 0.11$ & $7.74 \pm 0.75$ \\
\hline Positive & 4 & $4.17 \pm 0.89^{\prime \prime}$ & $0.40 \pm 0.07^{n}$ \\
\hline contro & 8 & $2.80 \pm 0.53^{x \pi}$ & $0.30 \pm 0.03^{\pi x}$ \\
\hline Metformin & 4 & $\begin{array}{c}1.88 \pm 0.57^{\pi \pi \Delta \Delta} \\
0.62+0.05^{\Delta \Delta}\end{array}$ & $\begin{array}{c}1.13 \pm 0.21^{\pi \pi} \Delta \\
188+0.36^{\pi \pi} \Delta \Delta\end{array}$ \\
\hline High & 4 & $0.85 \pm 0.17^{\Delta \Delta \Delta}$ & $0.96 \pm 0.16$ \\
\hline & 8 & $1.69 \pm 0.35^{\Delta \Delta \Delta \Delta}$ & $1.92 \pm 0.47^{\wedge \Delta \Delta}$ \\
\hline Low-dose & 4 & $1.44 \pm 0.41^{\pi \pi} \Delta \Delta$ & $0.90 \pm 0.19^{* \pi}$ \\
\hline UA & 8 & $0.82 \pm 0.20^{\Delta \Delta \# \#}$ & $2.32 \pm 0.48^{\star \pi \Delta \Delta}$ \\
\hline
\end{tabular}

$p<0.05$ and " $p<0.01$, compared to normal group; ${ }^{4} p$ $<0.05$ and ${ }^{\Delta \Delta} p<0.01$, compared to control group; ${ }^{\wedge} p$ $<0.05$ and ${ }^{\wedge} p_{<}<0.01$, compared to metformin group; ${ }^{\#} p<0.05$ and ${ }^{\# \#} p<0.01$, compared to high-dose UA group

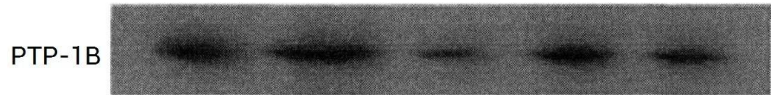

IRS-2

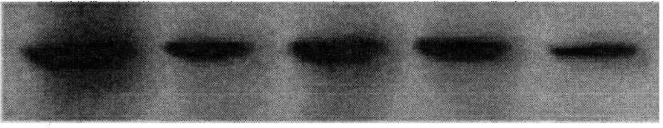

ACTB

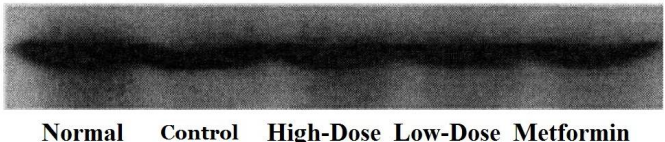

Figure 3: Effects of UA and metformin on the expressions of PTP-1B and IRS-2 proteins in rat liver at week $4(n=8)$

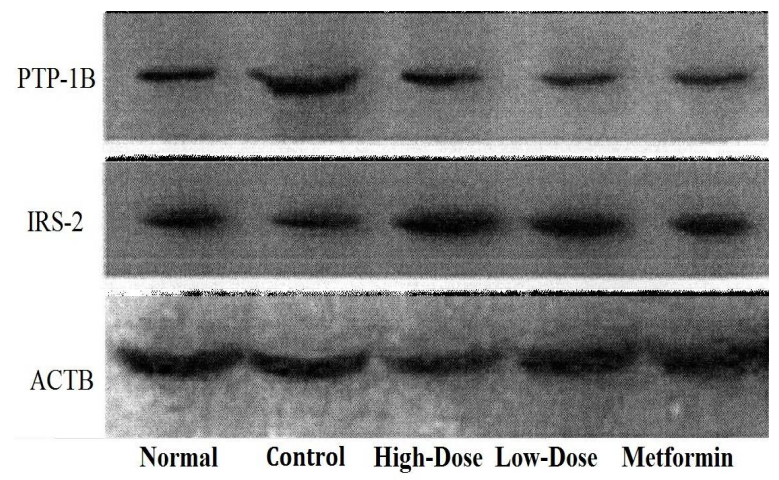

Figure 4: Effects of UA and metformin on the expressions of PTP-1B and IRS-2 protein in rat liver at week $8(n=8)$

\section{IRS-2 tyrosine phosphorylation in rat liver}

The results of western blot showed that IRS-2 tyrosine phosphorylation in liver tissue of rats in the control group was significantly lower than that of normal group. However, after 4 and 8 weeks of UA treatment, the phosphorylation of IRS-2 tyrosine increased. A similar effect was produced by metformin (Figure 5 and Figure 6 ). 


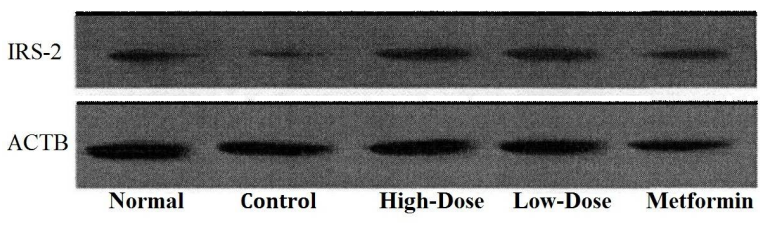

Figure 5: Effect of UA and metformin on IRS-2 tyrosine phosphorylation at week $4(n=8)$

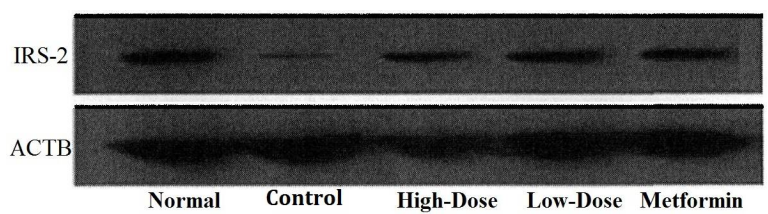

Figure 6: Effect of UA and metformin on IRS-2 tyrosine phosphorylation at week $8(n=8)$

\section{DISCUSSION}

The main features of IR are significant impairment of glucose metabolism and associated abnormal lipid metabolism. Insulin resistance increases gluconeogenesis and glycogen degradation, and decreases glycogen synthesis. At the same time, disorders in lipid metabolism result in excessive accumulation of fat in the liver parenchymal cells, ultimately leading to the occurrence of fatty liver [19]. Ursolic acid improves glucose tolerance and dyslipidemia [20]. Insulin tolerance test has shown that UA improves sensitivity to insulin, resulting in enhanced liver glycogen synthesis and mitigation of liver steatosis.

Insulin resistance is due to impairment of signal transduction to insulin receptor, which at the molecular level, involves IRS. This results in reduction in IRS gene expression, reduction in protein content, and dysfunction in insulin signaling. It is known that IRS-2 is present in the liver and islet $\beta$ cells where it regulates lipid synthesis through steroid regulatory element binding protein (SREBP). Down-regulation of IRS-2 affects the downstream signaling of $\mathrm{PI3K} / \mathrm{Akt}$, resulting in reduced hepatic glycogen synthesis and increased fat synthesis [21]. In the present study, IRS-2 mRNA and related protein expressions, and tyrosine phosphorylation in the liver of insulin-resistant rats were determined by molecular biology methods. The results obtained showed that the expressions of IRS-2 mRNA and its related proteins were significantly increased by UA, relative to the high fat diet-induced IR group (positive control). This finding suggests that reduced expression of IRS-2 may lead to IR.

PTP-1B interacts with the receptor for insulin as well as IRS. These interactions reduce the degree of tyrosine phosphorylation in these regulatory proteins, thereby blocking the insulin signal cascade. Reduction of the expression of PTP-1B in the liver and muscle of the diabetic animal model increases the level of tyrosine phosphorylation of insulin receptor and its substrate, which enhances insulin signaling and response [22]. In the present study, RT-PCR and Western blotting were applied in determining PTP-1B mRNA and its protein expressions in the liver of IR rats. Treatment with UA led to significantly lower expression of PTP-1B than in the untreated IR group. This indicates that the expression of PTP-1B may be one of the key molecular factors linked to insulin signal transduction and IR.

\section{CONCLUSION}

The results obtained in this study suggest that UA mitigates IR by inhibiting the expression of PTP-1B and up-regulating the expression of IRS2 mRNA. Thus, PTP-1B is a potential target for the treatment of type 2 diabetes.

\section{DECLARATIONS}

\section{Conflict of Interest}

No conflict of interest associated with this work.

\section{Contribution of Authors}

We declare that this work was done by the authors named in this article, and all liabilities pertaining to claims relating to the content of this article will be borne by them. All authors read and approved the manuscript for publication. Wang Ziwei conceived and designed the study, collected and analyzed the data, and wrote the manuscript.

\section{REFERENCES}

1. Balsan G, Vieira JL, Oliveira AM, Portal VL. Relationship between adiponectin, obesity and insulin resistance. Rev Assoc Med Bras 2015; 61: 72-80.

2. Adevaandany $M M$, Perezfelpete $N$, Fernandez-fernandez C, Donapetrygarcia C, Paz-osgarcia C. Liver glucose metabolism in humans. Bioscience Rep 2016; 36: e00416.

3. Rines $A K$, Sharabi $K$, Tavares $C D$, Puigserver $P$. Targeting hepatic glucose metabolism in the treatment of type 2 diabetes. Nat Rev Drug Discov 2016; 15: 786804.

4. Tilg $H$, Moschen AR, Roden M. NAFLD and diabetes mellitus. Nat Rev Gastro Hepat 2017; 14: 32-42.

5. Xu E, Schwab M, Marette A. Role of protein tyrosine phosphatases in the modulation of insulin signaling and 
their implication in the pathogenesis of obesity-linked insulin resistance. Rev Endocr Metab Dis 2013; 15: 7997.

6. Zheng $C$, Yang $Q, X u$ C, Shou $P$, Cao J, Jiang $M$, Chen Q, Cao G, Han Y, Li F, Cao W, Zhang L, Zhang L, Shi $Y$, Wang $Y$. CD11b regulates obesity-induced insulin resistance via limiting alternative activation and proliferation of adipose tissue macrophages. $P$ Natl Acad Sci USA 2015; 112: E7239-48.

7. Kashyap D, Tuli HS, Sharma AK. Ursolic acid (UA): A metabolite with promising therapeutic potential. Life Sci 2016; 201-213.

8. Castro AJG, Frederico MJS, Cazarolli LH, Mendes CP, Bretanha LC, Schmidt EC, Bouzon ZL, Pinto VADM, Ramos CDF, Pizzolatti MG, Silva FRMB. The mechanism of action of ursolic acid as insulin secretagogue and insulinomimetic is mediated by crosstalk between calcium and kinases to regulate glucose balance. BBA-Gen Subjects 2015; 1850: 51-61.

9. He Y, Li W, Li Y, Zhang S, Wang Y, Sun C. Ursolic acid increases glucose uptake through the pi3k signaling pathway in adipocytes. Plos One 2014; 9: e110711.

10. Stadlbauer $V$, Haselgrubler $R$, Lanzerstorfer $P$, Plochberger B, Borgmann D, Jacak J, Winkler SM, Schroder K, Hoglinger O, Weghuber J. Biomolecular characterization of putative antidiabetic herbal extracts. Plos One 2016; 11: e0148109.

11. Kunkel $S D$, Elmore CJ, Bongers KS, Ebert SM, Fox DK, Dyle MC, Bullard SA, Adams CM. Ursolic acid increases skeletal muscle and brown fat and decreases dietinduced obesity, glucose intolerance and fatty liver disease. Plos One 2012; 7: e39332.

12. Sundaresan A, Radhiga T, Pugalendi KV. Ursolic acid and rosiglitazone combination improves insulin sensitivity by increasing the skeletal muscle insulinstimulated IRS-1 tyrosine phosphorylation in high-fat diet-fed C57BL/6J mice. J Physiol Biochem 2016; 72: 345-352.

13. He Y, Li W, Li Y, Zhang S, Wang Y, Sun C. Ursolic acid increases glucose uptake through the pi3k signaling pathway in adipocytes. Plos One 2014; 9: e110711.

14. Ling $C$, Jinping L, Xia L, Yang R. Ursolic acid provides kidney protection in diabetic rats. Curr Ther Res Clin E 2013; 75: 59-63.

15. Kannappan S, Anuradha CV. Insulin sensitizing actions of fenugreek seed polyphenols, quercetin \& metformin in a rat model. Indian J Med Res 2009; 129: 401-408.

16. World Health Organization. Declaration of Helsinki. Br Med J 1996; 313: 1448-1449.

17. Matthews DR, Hosker JP, Rudenski AS, Naylor BA, Treacher DF, Turner RC. Homeostasis model assessment: Insulin resistance and beta-cell function from fasting plasma glucose and insulin concentrations in man. Diabetologia 1985; 28: 412-419.

18. Haffner SM, Gonzalez C, Miettinen H, Kennedy E, Stern MP. A prospective analysis of the HOMA model. The Mexico City Diabetes Study. Diabetes Care 1996; 19: 1138-1141.

19. Omar R, Yang J, Liu H, Davies DM, Gong Y. Hepatic stellate cells in liver fibrosis and siRNA-based therapy. Rev Physiol Bioch P 2016; 127: 1-37.

20. Kazmi I, Afzal M, Rahman S, Iqbal M, Imam F, Anwar F. Antiobesity potential of ursolic acid stearoyl glucoside by inhibiting pancreatic lipase. Eur J Pharmacol 2013; 709: 28-36.

21. Yao $H$, Han $X, \operatorname{Han} X$. The cardioprotection of the insulinmediated PI3K/Akt/mTOR signaling pathway. Am J Cardiovasc Drug 2014; 14: 433-442.

22. Bakke J, Haj FG. Protein-tyrosine phosphatase 1B substrates and metabolic regulation. Semin Cell Dev Biol 2015; 37: 58-65. 